\title{
A Novel Feature Points Description Algorithm based on Weighted Neighbor Contribution and Multi-resolution Fusion
}

\author{
Pei $\mathrm{Hu}^{1}$, Yanqing Shao ${ }^{1,2}$, Chaowei Tang ${ }^{1 *}$ and Siman Zhao ${ }^{3}$ \\ ${ }^{1}$ College of Communication Engineering, Chongqing University, Chongqing \\ 400044, China \\ ${ }^{2}$ Chongqing College of Electronic Engineering, Chongqing 401331, China \\ ${ }^{3}$ Patent Examination Cooperation Center of the Patent Office, SIPO, \\ Sichuan,610213, China \\ Corresponding author: Chaowei Tang [cwtang@cqu.edu.cn]
}

\begin{abstract}
Aiming at the long time consumption in the feature description, a feature points description algorithm based on weighted neighbor contribution and multi-resolution fusion is involved. First, the contribution of the sampling point's neighbors is introduced to design the Weighted Neighbor Contribution Center-Symmetric Local Binary Pattern (WNCCS-LBP) descriptor based on the weighted neighbor contribution strategy. Then, a Multi-resolution Fusion Center-Symmetric Local Binary Pattern(MFCS-LBP) descriptor is constructed by combining the WNCCS-LBP descriptor and the CS-LBP descriptor based on the multi-resolution fusion strategy. Experimental results demonstrate that the proposed algorithm significantly saves the description time and improve the robustness in the presence of scale change, image rotation, viewpoint change and image blur.
\end{abstract}

Keywords: Local feature description; SIFT; CS-LBP; Weighted neighbor contribution; Multi-resolution fusion

\section{Introduction}

Comparing with the global features such as variance and color histogram, local features are more robust to shade and interference and are widely used in image matching and target recognition etc.[1-4]. Furthermore, local feature descriptors are relatively insensitive to rotation, illumination and scale variations and they convert the image matching into the similarity measurement of feature vectors. An excellent local feature descriptor should be equipped with robustness and distinctiveness [5].

The two important steps to construct a descriptor are as follows. The first step is to localize the position and scale of the feature points by filtering the points that remain unstable over changes. The second step is to construct a description of the feature point. While the localization and description aspects of the feature points algorithm are often designed together, the solutions to these two problems are independent [6]. This paper focuses on the second aspect to explore description. Scale invariant feature transform(SIFT) [7] is one of the most widely used feature points description algorithm. SIFT is constructed by computing the direction of the feature point and counting the gradient magnitude and orientation of pixels around the feature point. SIFT is mostly invariance to scale change, image rotation and illumination. But the complexity of the construction process and high dimensionality of SIFT will lead to the low matching efficiency.

To solve these problems, many improved methods have been proposed and these approaches could be, at least roughly, classified into three classes. The first class includes scale invariant feature transform based on principal component analysis (PCA-SIFT) 
descriptor [6], boosting scale invariant feature transform (B-SIFT) descriptor [8] and improved SIFT [9]. They are presented to reduce the descriptor's dimensionality. PCA-SIFT and B-SIFT are the simplified version of the SIFT, which further deal with the gradient through PCA. The improved SIFT uses K-L transform to reduce the dimensionality of feature vector. Though the computing speed is enhanced with these methods, the performance of descriptor is influenced to some degree. The gradient location-orientation histogram [10] (GLOH) and the approach proposed by Zhou[11] belong to the second type which aims to change the shape of neighborhood. These techniques have less influence on algorithm performance. The GLOH descriptor is similar to SIFT and it also applies PCA to reduce the dimensionality of descriptor. Other methods such as PLBP[12], multi-support region ellipse-partition based gradient histogram(MEGH) [13] and center-symmetric local binary patterns (CS-LBP) descriptor [14] are the third category. The MEGH descriptor is greatly helpful to improve the computing speed in condition that the detected region is affine invariant and normalized. The dimensionality is moderate and the computing speed is fast for traditional CS-LBP descriptor, but the original $\mathrm{N}$ pixels in the neighborhood can't represent the local feature well. Furthermore, the CS-LBP has unsatisfying description performance because the CS-LBP algorithm ignores the contribution of other neighborhood pixels. What's more, the distinctiveness of the descriptor will decline when the gray values of the neighborhood pixels are close.

In view of the problems of SIFT and CS-LBP, we propose a feature points description algorithm based on weighted neighbor contribution and multi-resolution fusion, which can not only greatly reduce the description time but also improve the performance. The WNCCS-LBP descriptor is constructed based on the weighted neighbor contribution strategy which considers the contribution of the sampling point's neighbor. Then, the MFCS-LBP descriptor is constructed by combing the WNCCS-LBP descriptor and the CS-LBP descriptor to improve the distinctiveness and robustness of the description algorithm. This step is based on the multi-resolution fusion theory and the two descriptors WNCCS-LBP and CS-LBP are constructed under different sampling radius.

The rest of the paper is organized as follows. Section 2 reviews the CS-LBP descriptor and the multi-resolution fusion theory.The feature points description algorithm based on weighted neighbor contribution and multi-resolution fusion is detailed in Section 3. The performance of our scheme is reported in Section 4. The conclusion is given in Section 5.

\section{Review of the CS-LBP and Multi-resolution Fusion Theory}

\subsection{CS-LBP}

To reduce the high dimensionality of LBP, the CS-LBP [13] descriptor described by Marko Heikkilä employs binarization to the neighborhood of each pair pixels which are center symmetric as the central pixel. CS-LBP is defined as follows:

$$
\begin{aligned}
\operatorname{CS}-\operatorname{LBP}_{R, N, T}(u, v)=\sum_{i=0}^{N / 2-1} s\left(n_{i}-n_{i+N / 2}\right) 2^{i} \\
s(x)=\left\{\begin{array}{l}
1, x \geq T \\
0, \text { otherwise }
\end{array}\right.
\end{aligned}
$$

Where $n_{i}$ and $n_{i+N / 2}$ are the gray values of the pair pixels on a circle of radius $R$ and they are center symmetric as the central pixel $(u, v) . N$ is the number of equally spaced pixels on a circle of radius $R$. A relatively small value for $T$ should be utilized and the dimension of CS-LBP is $2^{N / 2}$. Experiments conducted by Marko Heikkilä [13] showed that the excellent matching was achieved when $N=8, R=2$ and $T=0.01$.

The CS-LBP descriptor not only possess a lower dimensionality but also has a better 
robustness in flat image area. However, the CS-LBP descriptor only compares the gray value of pair pixels which are center symmetric as the central pixel and ignores the contribution of other neighborhood pixels which makes the texture feature is not considered entirely.

\subsection{Multi-resolution Fusion Theory}

Multi-resolution fusion utilizes texture spectral histograms obtained under different resolutions (different $R$ ) to jointly present textural feature. This method firstly describes the neighborhoods under different resolutions to obtain the texture spectral histograms. Then, put the histograms together to present texture feature. It is possible to formulate the process as follows:

$$
\mathbf{S}=\left[\mathbf{s}_{1}, \mathbf{s}_{2}, \ldots \mathbf{s}_{i} \ldots, \mathbf{s}_{n}\right]
$$

Where $i$ denotes different resolution, $n$ is the number of resolutions, $\mathbf{s}_{i}(i=1,2, \ldots n)$ is the texture spectral histogram of different resolution and it is typically expressed as a vector. As a consequence, the generated descriptor $\mathbf{S}$ which considers the multi-resolution fusion strategy makes the region's information be more accurate, complete and abundant.

\section{Proposed Descriptor}

\subsection{WNCCS-LBP}

The CS-LBP description algorithm loses the contribution of the other neighborhood pixels and the original $\mathrm{N}$ neighborhood pixels isn't a good demonstration of texture information, in consequence the accuracy and completeness of the descriptor cannot be guaranteed. Aiming at the defects of CS-LBP, a weighted neighbor contribution strategy and a descriptor named WNCCS-LBP are explored. The WNCCS-LBP descriptor adopts alternately sampling and considers the ignored neighborhood pixels which have contributions in theory. The description process is as follows.

1) $2 N$ neighborhood pixels are given by $(-R \sin (2 \pi j / 2 N), R \cos (2 \pi j / 2 N)$, $(j=1,2, \ldots \ldots .2 N)$ and the gray values of neighborhood pixels which are not in the image grids are estimated by bilinear interpolation.

2) $N$ equally spaced sampling points on a circle of radius $R$ are obtained through alternately sampling.

3) Seek the contribution of sampling point's neighbors and then average the gray value of sampling points and the contribution previously stated. So far, the weighted gray values of sampling points are acquired.

4) The binarization of the neighborhood of each weighted pair pixels which are center symmetric as the central pixel is implemented.

The encoding rules of WNCCS-LBP are defined as follows:

$$
\begin{gathered}
{\operatorname{WNCCS}-L_{R B}}_{R, N T}(u, v)=\sum_{i=0}^{N / 2-1} s\left(\frac{w\left(m_{i}+m_{i+1}\right)+n_{i}}{2 w+1}-\frac{w\left(m_{i+N / 2}+m_{i+N / 2+1}\right)+n_{i+N / 2}}{2 w+1}\right) 2^{i} \\
s(x)=\left\{\begin{array}{l}
1, x \geq T \\
0, \text { otherwise }
\end{array}\right.
\end{gathered}
$$

Where $n_{i}$ and $n_{i+N / 2}$ are the gray values of sampling points. . $w$ is the weight of the skipped neighborhood pixels to the central point and $T$ is the threshold. $m_{i}$ and $m_{i+1}$ are the sampling point's neighbor which are adjacent to $n_{i}$. Especially, $m_{i+N / 2+1}$ equal to $m_{0}$ when $i=N / 2-1$. Since each sampling point's neighbor is adjacent to the two sampling points and the contribution of it only affects the two sampling points. So the 
contributions to these two sampling points are equal and $w=0.5 . w\left(m_{i}+m_{i+1}\right)$ and $w\left(m_{i+N / 2}+m_{i+N / 2+1}\right)$ are the contribution of sampling point's neighbor. $\left[w\left(m_{i}+m_{i+1}\right)+n_{i}\right] /(2 w+1)$ and $\left[w\left(m_{i+N / 2}+m_{i+N / 2+1}\right)+n_{i+N / 2}\right] /(2 w+1)$ are the weighted gray values of sampling points. The encoding rule of CS-LBP and WNCCS-LBP descriptor is illustrated in Table 1, where all points on the circle of radius $R$ are neighborhood pixels. The points with a circular border are the sampling points and the rest with rectangular border are the sampling point's neighbors also called the skipped neighborhood pixels. In order to increase the persuasiveness of Table 1, the threshold $T$ is 5 which is different from the value in our following experiments. Table 1 shows that CS-LBP lead to the undesirable coded values in both non-flat image regions and flat image regions. On the contrary, WNCCS-LBP achieves the satisfied and distinguishable results because of the contribution of the skipped neighborhood pixels which improve the distinctiveness of descriptor.

Table 1. The Schematic Diagram of Two Encoding Rules $(T=5)$

\begin{tabular}{ccccccc}
\hline Local Image Region & The Result of CS-LBP & The Result of \\
WNCCS-LBP
\end{tabular}

\subsection{MFCS-LBP}

The structural characteristics of an image are uncertain, so it is insufficient to represent texture information of an image accurately with a single texture descriptors. Furthermore, the adjacent texture descriptors are not completely independent from each other and each texture primitive may limit or affect adjacent texture primitive, which makes the "effective area" of a descriptors be slightly larger than the scope of the original description. So for the same region, the CS-LBP descriptors with different spatial scales contain different information.

In view of the above situations, a multi-resolution fusion strategy which combines the WNCCS-LBP descriptor and the CS-LBP descriptor is presented to further improve the accuracy and completeness of the descriptors. The two generated texture spectral histograms of CS-LBP and WNCCS-LBP are as follows: 


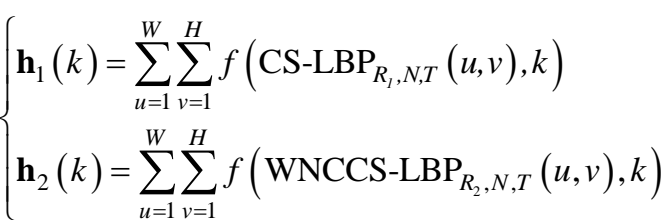

$$
\begin{aligned}
& f(x, y)=\left\{\begin{array}{l}
1, x=y \\
0, \text { otherwise }
\end{array}\right.
\end{aligned}
$$

Where $k \in[0, K]$ and $K$ is the maximum encoded value of CS-LBP or WNCCS-LBP. $W$ and $H$ are the width and height of feature region respectively. The MFCS-LBP descriptor $\mathbf{H}_{\text {MFCS-LBP }}$ is derived by combing the two generated texture spectral histograms according to equation (5):

$$
\begin{aligned}
& \mathbf{H}_{\text {MFCS-LBP }}=\left[\alpha \mathbf{h}_{1}, \beta \mathbf{h}_{2}\right] \\
& \alpha=\frac{\sum_{j=1}^{m} p_{1}^{j} \lg p_{1}^{j}}{\sum_{j=1}^{m}\left(p_{1}^{j} \lg p_{1}^{j}+p_{2}^{j} \lg p_{2}^{j}\right)}, \beta=1-\alpha
\end{aligned}
$$

Where $\alpha, \beta \in[0,1]$ are the weights for two histograms. $-\sum_{j=1}^{m} p_{1}^{j} \lg p_{1}^{j}$ is the information entropy which is proportional to the weight. The value of $m$ is 256 and $p_{1}^{j}$ denotes the number of $j$-level pixels in the circle of radius $R_{1}$.

In summary, the calculation of MFCS-LBP descriptor is as follows.

1) For each pixel in the feature region, calculate $\mathrm{CS}-\mathrm{LBP}_{R_{1}, N, T}(u, v)$ with $R_{1}=1, N=8, T=0.01$ according to equation (1) and $\mathrm{WNCCS}-\operatorname{LBP}_{R_{2}, N, T}(u, v)$ with $R_{2}=2, N=8, T=0.01$ according to equation (3).

2) Calculate the two texture spectral histograms: $\mathbf{h}_{1}$ and $\mathbf{h}_{2}$ according to equation (4).

3) Combine $\mathbf{h}_{\mathbf{1}}$ and $\mathbf{h}_{\mathbf{2}}$ according to equation (5) to build $\mathbf{H}_{\mathrm{MFCS}-\mathrm{LBP}}$ which is the MFCS-LBP descriptor of the feature region. In conclusion, the construction of the proposed feature points description algorithm is as follows. The SIFT algorithm [6] is utilized to localize features point in position and scale and assign an orientation to each feature point to get the rotational invariance. Firstly, the $16 \times 16$-pixel feature regions that centered on one feature point are delimited and the gradient magnitude and orientation of each pixel in the region are calculated. Secondly, the orientation histograms are formed by counting these gradient orientations. Each orientation histogram has 36 bins covering the 360 degree range of orientations and each point added to the histogram is weighted by its gradient magnitude and Gaussian-weighted circular window. Then, the orientation of peak in the orientation histogram is regarded as the dominant orientation of the feature point. Any other local peaks that are within $80 \%$ of the highest peaks are regarded as the auxiliary directions. Finally, the rotational invariance is achieved by rotating coordinate axis to dominant direction.

Once the rotating is completed, the $16 \times 16$-pixel feature region is divided into 16 sub-regions $\operatorname{bin}_{i}(i=1,2, \ldots \ldots . .16)$ with size of $4 \times 4$ in order to indicate the spatial property. Then calculate the MFCS-LBP descriptors $\mathbf{H}_{\mathbf{1}}, \mathbf{H}_{\mathbf{2}} \ldots . . . \mathbf{H}_{\mathbf{1 6}}$ for each sub-region according to chapter three. The sub-regions which are closer to the feature point have greater contribution to feature point, so let's start from the closer feature point and put 
$\mathbf{H}_{1}, \mathbf{H}_{2} \ldots \ldots \mathbf{H}_{16}$ together from inside to outside to describe the $16 \times 16$-pixel feature region. Finally, the description vector $\mathbf{F}=\left[\mathbf{H}_{1}, \mathbf{H}_{2}, \ldots . . \mathbf{H}_{16}\right]$ is obtained.

Finally, in order to increase the robustness of feature vector to illumination, we can learn from SIFT algorithm and normalize the description vector twice [6]. Normalizing the feature vector $\mathbf{F}$ to unit length for the first time aims to eliminate the influence of linear illumination. Then the elements of the unit feature vector which are greater than 0.2 are assigned the value 0.2 to reduce the influence of large gradient magnitudes. At last, normalizing for the second time aims at nonlinear illumination.

\section{Experiments and Analyses}

\subsection{Image Matching}

In the experiment, Mikolajczyk [10] dataset is adopted for the simulation. The test data includes six kinds of geometric and photometric transformations such as scale change, image rotation, viewpoint change, illumination change, image blur and JPEG compression. The matching performance of the proposed algorithm in complex environment can be checked on this image set. Five pairs of images selected from the image set are shown in Figure 1.

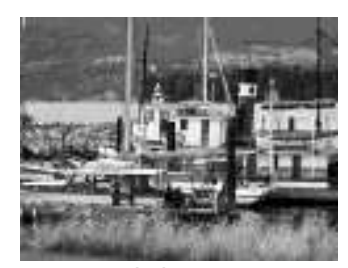

(a) scale change + image rotation

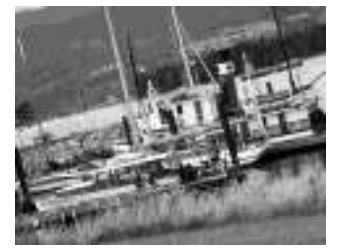

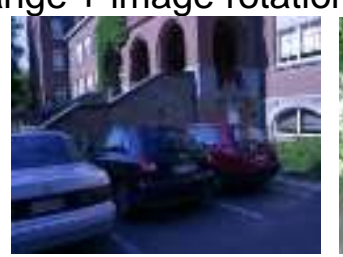

(c) illumination change

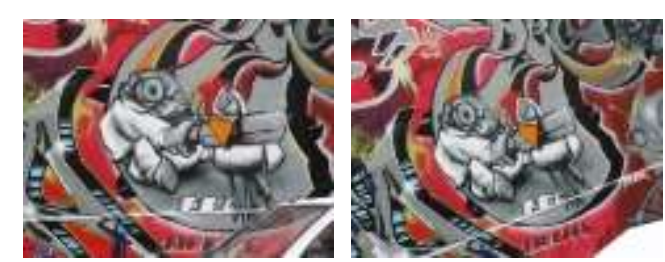

(b) viewpoint change
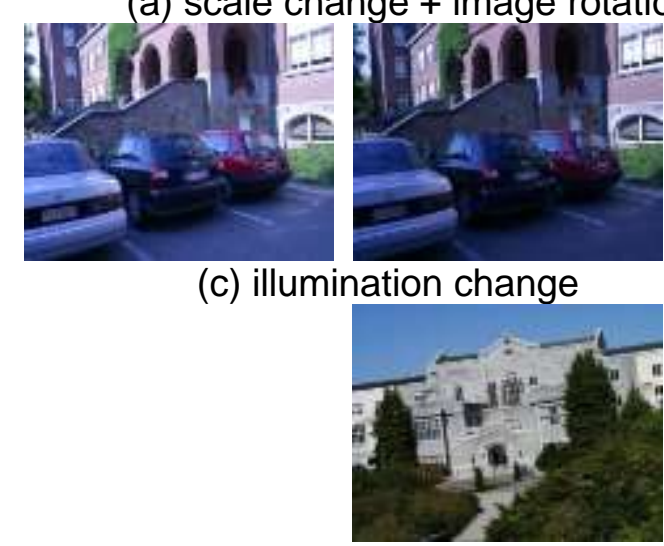

(e) JPEG compression

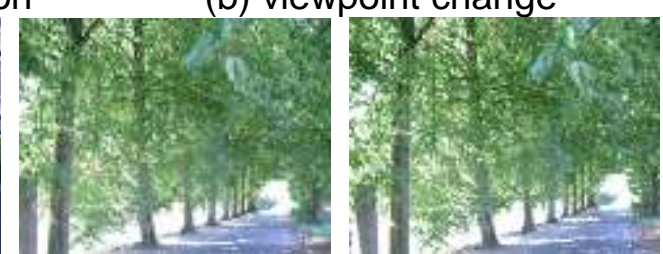

(d)image blur

Figure 1. Testing Image Pairs

Firstly, the SIFT algorithm is adopted to extract the feature points from five pairs of images, and the proposed algorithm is exploited for describing the extracted feature points. Cosine distance is used to measure the similarity of the feature vector and the nearest neighbor rule is used for matching. If the cosine distance of two feature vectors for a pair of feature points falls below the chosen threshold, this pair is considered to be matched. This paper presents recall vs. 1 - precision graphs to measure matching performance by varying the threshold, where recall and precision are define as follows: 
recall $=\frac{\# \text { correct } \text { matches }}{\# \text { correspondences }} \times 100 \%$

precision $=\frac{\# \text { correct matches }}{\text { \#all matches }} \times 100 \%$

The algorithms are implemented in MATLAB R2009b and run on a PC with Intel Core i7-3770 processor, $3.4 \mathrm{GHz}$ with 4GB RAM. The effectiveness of the proposed algorithm is verified by comparing it with SIFT and CS-LBP. Table 2 lists the construction time of descriptors and figure 2 shows the matching performance comparison of the three algorithms

Table 2. Construction Time of Three Kinds of Descriptors(s)

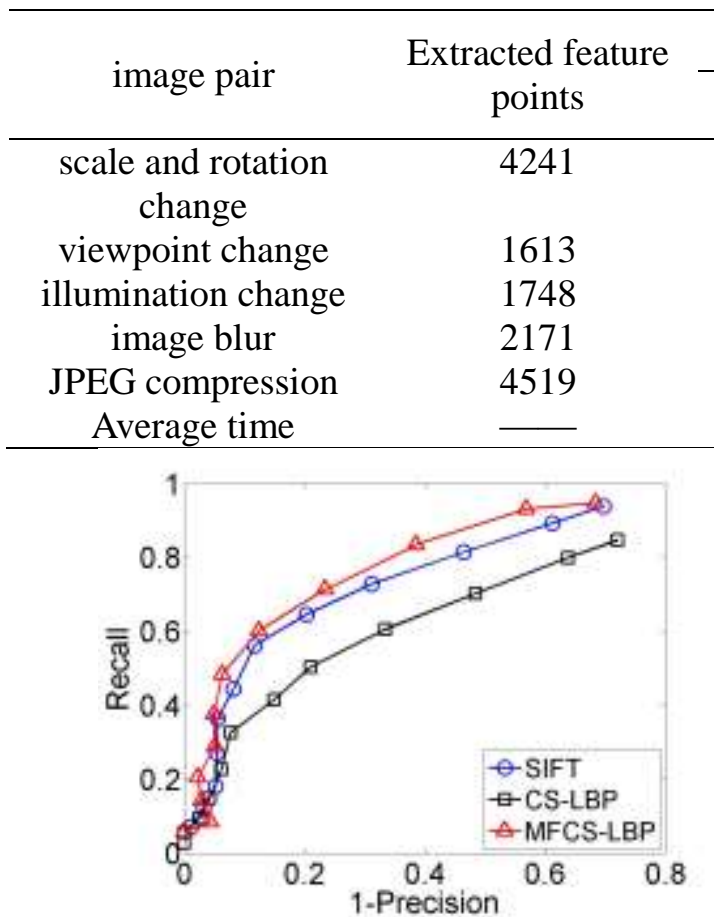

(a) Scale change + image rotation

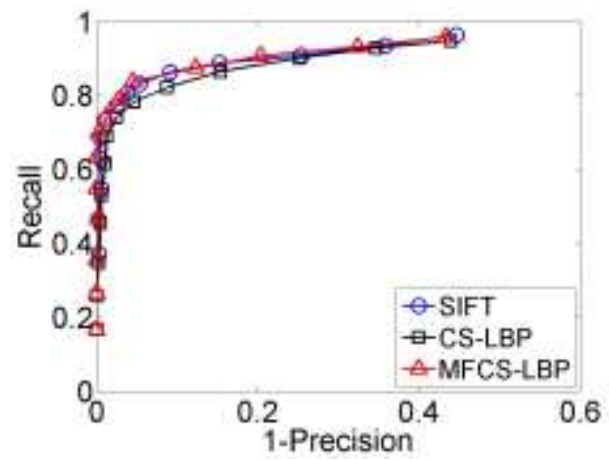

(c) Illumination change construction time

\begin{tabular}{ccc} 
SIFT & CS-LBP & MFCS-LBP \\
133.53 & 14.21 & 28.34 \\
& & \\
50.74 & 3.77 & 8.41 \\
49.71 & 2.41 & 6.28 \\
87.52 & 6.73 & 15.32 \\
125.38 & 12.46 & 27.66 \\
87.85 & 9.68 & 16.67 \\
\hline
\end{tabular}

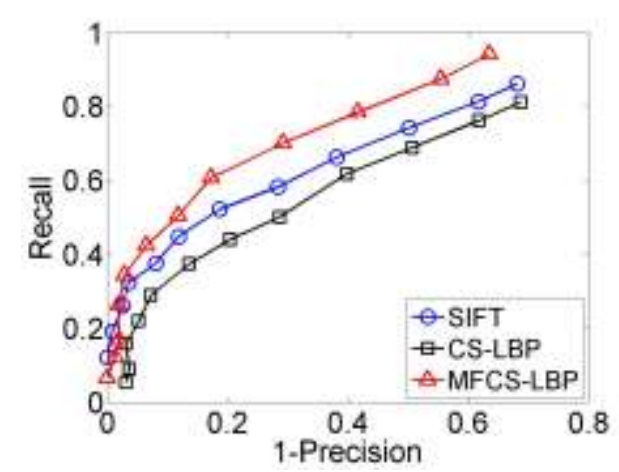

(b) Viewpoint change

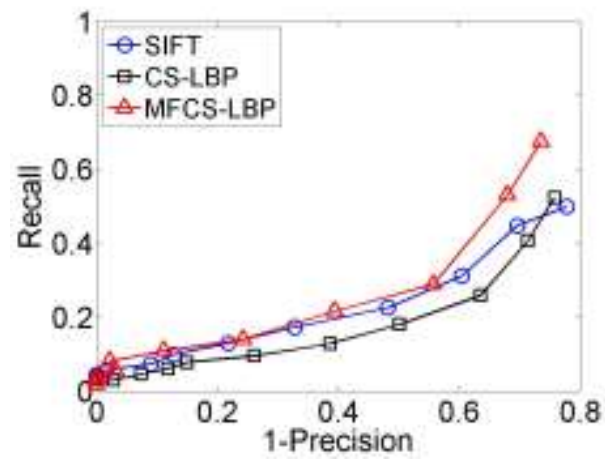

(d) Image blur 


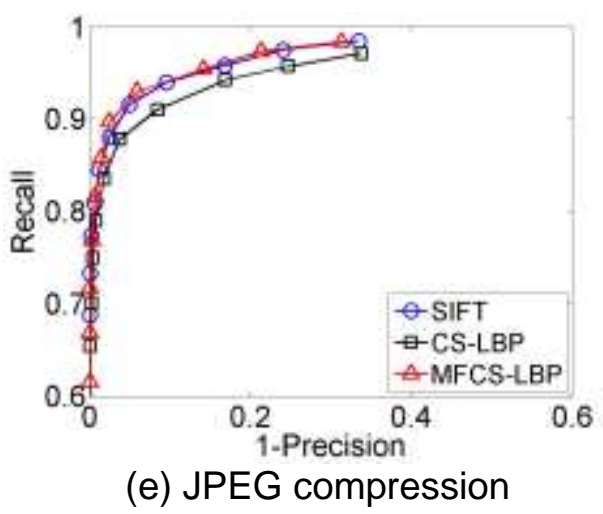

Figure 2. The Matching Performance of the Three Algorithms

Table 2 shows that the construction time of CS-LBP and MFCS-LBP is far below than that of SIFT. The SIFT descriptor is constructed by distributing the weight of each pixel into adjacent histogram bins, which means that each weight is shared between 8 bins. However, CS-LBP value is quantized by its nature, thus each weight is shared between 4 bins. What's more, the SIFT descriptor which uses gradient as the local feature requires more time to calculate the gradient orientation while LBP descriptor needs only simple arithmetic operations. Therefore, the feature description algorithm introduced into the LBP can reduce the description time greatly.

As is shown in Figure 2(a) and Figure 2(b), the proposed MFCS-LBP descriptor has superior matching performance in the presence of image stretching. For the proposed algorithm, the multi-resolution fusion strategy provides more texture information by introducing additional descriptors constructed under small radius. Furthermore, the weighted neighbor contribution strategy considers the information of the sampling point's neighbor, which allows the neighborhood information more comprehensive and improves the universality. In the case of Figure 2(d), the MFCS-LBP descriptor still performs obvious advantages when the images become blurred and the distinctiveness of local region decreases. The reason is that weighted neighbor contribution strategy can greatly promote the diversity of different regions thereby enhance the distinctiveness of feature descriptor. However, as shown in Figure 2(c) and 2(e), the presented algorithm has unsatisfied performance to illumination and JPEG compression with respect to the first three transformations. This could be explained by the fact that we adopt the same method to eliminate illumination influence compared to SIFT and CS-LBP. But we should note that the construction time of MFCS-LBP is one fifth of SIFT. In summary, the feature points description algorithm not only saves the description time but also exhibits the best robustness and distinctiveness among three algorithms

\subsection{Image Retrieval}

We enhanced the Corel database [15] to evaluate the proposed descriptor. Corel image library contains ten types of images and each type includes one hundred images. We first select ten images from each type randomly. Then the scale and rotation transformations in three different extents are done for each selected image. The original image and the three transformed images constitute a sub-type. Therefore, the newly-built sub-database includes four hundred images. Similarly, select another ten images from each type randomly and conduct viewpoint change, image blur, illumination change and JPEG compression for each image. So far, the five sub-databases are constructed. SIFT, CS-BP and the proposed MFCS-LBP description algorithms will be performed on these five sub-databases to compare their retrieval performance for different transformations.

The evaluation criterion is based on the matching correctness. In experiment, 10 
images randomly selected from one sub-database are used as retrieval images and the rest are candidate images. For each retrieval image the output contains ten images which are most similar to the retrieval image. For each enhanced sub-database, 100 images will be retrieved. The specific method is as follow:

$$
R A=\frac{2 p+q}{13} \times 100 \%
$$

Initial retrieve score is set to 0 . If the ten selected best-match images contain $p$ images which belong to the same sub-type with the retrieval image, add $2 p$ points to the retrieves score. However, if $q$ images belong to the same type with retrieval images, add $q$ points. Unfortunately, if there is no qualified image in ten selected best-match images, the retrieves score remains unchanged. For each retrieved image, the ten selected best-match images include no more than three images that belong to the same sub-type with the retrieved image, so the correct retrieval score is $13(2 \times 3+1 \times 7=13) . R A$ represents the retrieval accuracy.

The result is shown in Table 3. Where (1) -(5) represents sub-database which contains scale change and image rotation, viewpoint change, illumination change, image blur and JPEG compression respectively. Different algorithms show various retrieval performances on different sub-databases. For some sub-databases which contain images with viewpoint change, scale change and image rotation, the proposed algorithm outperforms the SIFT and the CS-LBP, which is consistent with the matching results. The construction time of MFCS-LBP is almost $42 \%$ up compared with CS-LBP from Table 2 but the performance of image retrieval of MFCS-LBP increase 3.24\% compared with CS-LBP. What's more, the construction time of MFCS-LBP is one fifth of SIFT and the matching correctness of MFCS-LBP increase $1.71 \%$ compared with SIFT. Therefore, the feature points description algorithm combining weighted neighbor contribution with multi-resolution fusion gets a best performance compared with CS-LBP and SIFT.

\section{Table 3. The Retrieval Performance of Three Kinds of Algorithms on 5 different Sub-databases (\%)}

\begin{tabular}{ccccccc}
\hline \multicolumn{7}{c}{ sub-database } \\
\hline algorithm & $(1)$ & $(2)$ & $(3)$ & $(4)$ & $(5)$ & average \\
SIFT & 57.67 & 56.33 & 60.72 & 42.19 & 60.34 & 55.45 \\
CS-LBP & 55.49 & 54.67 & 59.34 & 40.21 & 59.87 & 53.92 \\
MFCS-L & 60.33 & 59.67 & 60.88 & 44.13 & 60.79 & 57.16 \\
BP & & & & & & \\
\hline
\end{tabular}

\section{Conclusion}

A new descriptor MFCS-LBP is presented by combining the WNCCS-LBP descriptor and the CS-LBP descriptor. The presented WNCCS-LBP descriptor which makes the information of the feature region be more complete and accurate is based on weighted neighbor contribution strategy. The multi-resolution fusion strategy consolidates the stability of feature regions under different resolution. The new MFCS-LBP descriptor is constructed to improve the tolerance to viewpoint change, scale change and so on. The performance of the proposed algorithms is compared with SIFT and CS-LBP. The comparisons of the performance among these algorithms include construction time, image matching and image retrieval. The experimental results show that the robustness of the proposed technique against scale change, image rotation, viewpoint change and image blur. 


\section{Acknowledgements}

This work was supported by the Special Fund of Chongqing Science and Technology Commission (NO. cstc2013shmszx0500)

\section{References}

[1] S. Saleem and R. Sablatnig, "A Robust SIFT Descriptor for Multispectral Images", IEEE signal processingletters, vol.21, no.4, (2014), pp. 400-403.

[2] X. Q. Wu and Q. S. Zhao, W. Bu, "FT-based contactless palmprint verification approach using iterative RANSAC and local palmprint descriptors", Pattern Recognition: The Journal of the Pattern Recognition Society, vol.47,no.10,(2014),pp. 3314-3326.

[3] C. Singh, N. Mittal and E. Walia, "plementary feature sets for optimal face recognition". EURASIP Journal on Image and Video Processing, vol.2014, no.1, (2014), pp. 1-18.

[4] A. Sedaghat and H. Ebadi, "Remote Sensing Image Matching Based on Adaptive Binning SIFT Descriptor", IEEE Transactions on Geoscience and Remote Sensing,vol.53,no.10,(2015),pp.5238-5239.

[5] P. Sykora, P. Kamencay and R. Hudec, "Comparison of SIFT and SURF Methods for Use on Hand Gesture Recognition based on Depth Map", AASRI Procedia, vol.9 (2014), pp.19-24.

[6] K. Yan and R. Sukthankar, "PCA-SIFT: a more distinctive representation for local image descriptors", Proceedings of the IEEE Conference on Computer Vision and Pattern Recognition. Washington, DC, United states, (2004) June 27- July 2.

[7] D. G. Lowe, "Distinctive image features from scale-invariant keypoints", International Journal of Computer Vision, vol.60, no.2, (2004), pp. 91-110.

[8] F. Mehran, K. Shohreh, M. S. Ehsan and F. Karim, "BSIFT: Boosting SIFT Using Principal Component Analysis", 22nd Iranian Conference on Electrical Engineering, Tehran, Iran, (2014) May 20-22.

[9] J. Zhao, T. Xin and G. Men, "Improved SIFT features in image retrieval using", 2011 3rd International Conference on Computer Research and Development (ICCRD), (2011) March 11-13.

[10] K. Mikolajczyk and C. Schmid, "A performance evaluation of local descriptors", IEEE Transactions on Pattern Analysis and Machine Intelligence, vol.27, no.10, (2005), pp.1615-1630.

[11] W. Zhou, C. Wang, B. Xiao and Z. Zhang, "SLD: A Novel Robust Descriptor for Image Matching", IEEE Signal Processing Letters, vol.3, no.21, (2014), pp.339-342.

[12] X. Qian, X. S. Hua, P. Chen and L. Ke, "PLBP: An effective local binary patterns texture descriptor with pyramid representation", Pattern Recognition, vol.44, no.10-11, (2011), pp.2502-2515.

[13] X. J. Dong, E. Liu, J. Yang and Q. Wu, "MEGH: A New Affine Invariant Descriptor", KSII Transactions on Internet and Information Systems, vol.7, no.7, (2013), pp.1690-1704.

[14] M. Heikkilä, M. Pietikäinen and C. Schmid, "Description of interest regions with local binary patterns", Pattern Recognition, vol.42, no.3, (2009), pp. 425-436.

[15] Image Dataset [DB/OL], http://wang.ist.psu.edu/docs/related/.
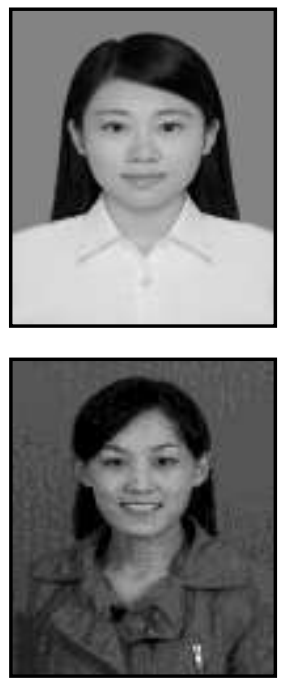

\section{Authors}

Pei Hu, she received the bachelor's degree in Electronical Information Science and Technology from Sichuan University of Science \& Engineering. She is currently studying for a master's degree at College of Communication Engineering, Chongqing University. The current research interests include image processing and intelligent information processing.

Yanqing Shao, she received her master's degree in 2011 from Chongqing University, currently studying on her Ph.D in College of Communication Engineering, Chongqing University and working in Chongqing College of Electronic Engineering. Her research topics are image processing and pattern recognition. 


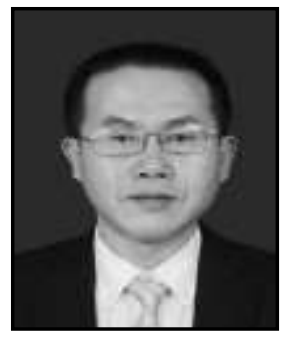

Chaowei Tang, he received his Ph.D. degree in 1993 from Chongqing University. In 2010, he went to Australia to study at the University of Adelaide. Currently he is a professor in Chongqing College of Electronic Engineering, Chongqing University. His research interests are digital image processing technology and intelligent information processing.

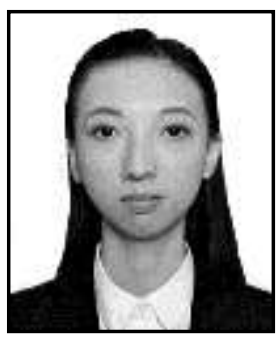

Siman Zhao, she received her master's degree in 2015 from Chongqing College of Electronic Engineering ,Chongqing University. She is currently working in Patent Examination Cooperation Center of the Patent 0ffice.She is currently researching on Multimedia Information Processing and computer vision. 
International Journal of Signal Processing, Image Processing and Pattern Recognition Vol. 10, No. 6 (2017) 